\title{
Fragilidade em Idosos Brasileiros - FIBRA-RJ: metodologia de pesquisa dos estudos de fragilidade, distúrbios cognitivos e sarcopenia
}

\author{
Roberto A. Lourenço, ${ }^{1,2 *}$ Maria Angélica Sanchez, ${ }^{1,2}$ Virgílio G. Moreira, ${ }^{1,2}$ Pricila Cristina C. Ribeiro, ${ }^{1,3}$ \\ Mariângela Perez, ${ }^{1,2}$ Glaucia Cristina Campos, ${ }^{1,2}$ Ismênia Blavatsky, ${ }^{1,4}$ Alina L. G. Teldeschii ${ }^{1,2}$
}

\section{Resumo}

A síndrome de fragilidade está associada a eventos indesejáveis, como incapacidade e morte. No Brasil, é uma condição pouco conhecida. O objetivo do presente relato é apresentar a metodologia de trabalho utilizada no FIBRA-RJ, seção Rio de Janeiro do estudo Fragilidade em Idosos Brasileiros (FIBRA-BR), desenvolvido para superar esta lacuna. A linha de base (primeira fase) do estudo FIBRA-RJ foi desenvolvida em 2009-2010. A amostra (847 indivíduos, $\geq 65$ anos, clientes de uma operadora de saúde) foi aleatória, estratificada por sexo e idade. Teve como objetivo estimar a prevalência de fragilidade e seus fatores associados. A segunda fase (2010-2011) teve como objetivo estimar a prevalência da demência e seus fatores associados. A terceira fase (2012-2013) buscou estimar a prevalência e incidência de sarcopenia/obesidade sarcopênica e seus fatores associados. A prevalência de fragilidade foi de 9,1\%; esteve associada a idade, menores escolaridade e cognição, percepção de saúde negativa, comorbidades e pior funcionalidade. A prevalência de demência foi de 16,9\%; esteve associada a idade e analfabetismo. No total, foram publicados onze artigos, quatro dissertações e duas teses; outras sete dissertações e teses estão em andamento, incluindo resultados da terceira fase. O FIBRA-RJ tem relatado estudos descritivos com conhecida limitação associada à causalidade reversa. $\mathrm{A}$ fase 3 nos permitirá estudar incidências e validade preditiva de biomarcadores vis-à-vis mortalidade, desfechos adversos e uso de serviços de saúde. O FIBRA-RJ representa um grande avanço no conhecimento sobre os agravos de saúde que acometem a população idosa brasileira.

Descritores: Vulnerabilidade; Idoso frágil; Estudos epidemiológicos; Envelhecimento.

\section{Abstract \\ Frailty in Older Brazilians - FIBRA-RJ: research methodology on frailty, cognitive disorders and sarcopenia}

The frailty syndrome is associated with adverse events such as disability and death. In Brazil, it is a poorly understood condition. The objective of this report is to present the methodology used in FIBRA-RJ, the Rio de Janeiro section of the study Frailty in the Brazilian Older (FIBRA-BR), developed to overcome this gap. The baseline (phase 1) study was developed in 2009-2010. The sample (847 individuals, $\geq 65$ years, customers from a health provider) was randomly selected, and stratified by sex
1. Laboratório de Pesquisa em Envelhecimento Humano. Universidade do Estado do Rio de Janeiro. Rio de Janeiro, RJ, Brasil.

2. Faculdade de Ciências Médicas. Departamento de Medicina Interna. Universidade do Estado do Rio de Janeiro. Rio de Janeiro, RJ, Brasil.

3. Departamento de Psicologia. Faculdade de Filosofia e Ciências Humanas. Universidade Federal de Minas Gerais. Belo Horizonte, MG, Brasil.

4. Fundação Instituto Brasileiro de Geografia e Estatística (IBGE), RN Brasil.

\author{
*Endereço para correspondência: \\ GeronLab, UER」 \\ Av. Marechal Rondon, 381, 20 andar \\ Rio de Janeiro, RJ, Brasil. CEP: 20950-000 \\ E-mail: lourenco@uerj.br
}

Revista HUPE, Rio de Janeiro, 2015;14(4):13-23

doi: $10.12957 /$ rhupe.2015.20066

Recebido em 04/12/2015. Aprovado em 08/12/2015.

and age. The study estimated the prevalence of frailty and its associated factors. The second phase (2010-2011) aimed to estimate the prevalence of dementia and its associated factors. The third phase (2012-2013) estimated the prevalence and incidence of sarcopenia/sarcopenic obesity and its associated factors. The prevalence of frailty was 9.1\%; it was associated with age, lower education and cognition, negative health perception, comorbidities and worse functionality. The prevalence of dementia was $16.9 \%$; it was associated with age and illiteracy. In total, eleven articles, four dissertations and two theses were published; seven other theses and dissertations are in progress, and they will include the results of phase 3 . The FIBRA-RJ has reported descriptive studies with well-known limitations associated with reverse causality. The phase 3 will enable us to study incidences, and predictive validity of biomarkers vis-à-vis mortality, adverse health outcomes and use of health services. The FIBRA-RJ study represents an advance in understanding the health complaints that affect Brazilian elderly.

Keywords: Vulnerability; Frail elderly; Epidemiologic studies; Aging. 


\section{Artigo original}

\section{Resumen}

Fragilidad en Ancianos brasileños - FIBRA-RJ: metodología de investigación de los estudios de fragilidad, trastornos cognitivos y sarcopeni

El síndrome de fragilidad está asociada con eventos adversos como discapacidad y muerte. En Brasil, es una enfermedad poco conocida. El objetivo de este informe es presentar la metodología de trabajo utilizada en FIBRA-RJ, sección Río de Janeiro del estudio Fragilidad en Adultos Mayores Brasileños (FIBRA-BR), desarrollado para superar esta deficiencia. La línea de base (fase 1) del estudio de FIBRA-RJ se desarrolló en 2009-2010. La muestra (847 individuos, $\geq 65$ años, clientes de un proveedor de salud) fue aleatoria, estratificada por sexo y edad. El objetivo fue estimar la prevalencia de fragilidad y sus factores asociados. La segunda fase (2010-2011) tuvo como objetivo estimar la prevalencia de demencia y sus factores asociados. La tercera fase (2012-2013) tuvo como objetivo

\section{Introdução}

A síndrome de fragilidade do idoso é uma condição clínica secundária à redução da reserva funcional de múltiplos sistemas fisiológicos tendo como consequência elevado risco de incapacidade e morte, após mínima exposição a fatores estressantes., Esta condição está diretamente associada a eventos indesejáveis como quedas, infecções, morbidades e institucionalização. ${ }^{3-5}$ Em 2001, Fried e colaboradores, propuseram uma definição operacional da síndrome de fragilidade em idosos. ${ }^{2}$

Em 2006, com o objetivo de descrever a prevalência de fragilidade e seus fatores associados, um grupo de pesquisadores de universidades brasileiras propôs uma rede de cooperação, o estudo Fragilidade em Idosos Brasileiros (FIBRA-BR). Ele foi idealizado por um consórcio de quatro universidades (Universidade do Estado do Rio de Janeiro, Universidade de São Paulo-Ribeirão Preto, Universidade Federal de Minas Gerais e Universidade Estadual de Campinas) e o financiamento de suas atividades de investigação foi aprovado no final de 2006, pelo Conselho Nacional de Desenvolvimento Científico e Tecnológico (CNPq), processo número 555087/20069. O FIBRA-BR seguiu um delineamento transversal, observacional e multicêntrico. Entre os anos de 2009 e 2010, entrevistou e examinou indivíduos com 65 anos ou mais, moradores na comunidade, em cidades de diferentes regiões do país, com distintas características sociodemográficas. As cidades participantes do estudo foram escolhidas segundo a conveniência dos coordenadores da pesquisa; no entanto, a amostra em cada cidade foi selecionada por uma estratégia proba- estimar la prevalencia e incidencia de la sarcopenia/obesidad sarcopénica y sus factores asociados. La prevalencia de fragilidad fue del 9,1\%; se asoció con edad, educación y cognición, percepción negativa de la salud, comorbilidad y peor funcionalidad. La prevalencia de demencia fue de 16,9\%; se asoció con edade y analfabetismo. En total, se publicaron: once artículos, cuatro disertaciones y dos tesis; otras siete disertaciones y tesis están en marcha, incluyendo resultados de la tercera fase. El FIBRA-RJ ha reportado estudios descriptivos con limitaciones conocidas asociadas con causalidad reversa. La fase 3 nos permitirá estudiar las incidencias y la validez predictiva de biomarcadores con respecto a la mortalidad, desenlaces adversos y uso de servicios de salud. El estudio FIBRA-RJ representa un gran avance en la comprensión de los problemas de salud que afectan a la población de adultos mayores brasileños.

Palabras clave: Vulnerabilidad; Adultos mayores frágiles; Estudios epidemiológicos; Envejecimiento.

bilística, estratificando os indivíduos por sexo e faixa etária. No total, participaram do estudo 7.609 idosos, distribuídos em 16 municípios - Barueri, SP; Belém, PA; Belo Horizonte, MG; Campina Grande, PB; Campinas, SP; Cuiabá, MT; São Paulo, SP; Fortaleza, CE; Ivoti, RS; Parnaíba, PI; Poços de Caldas, MG; Recife, PE; Ribeirão Preto, SP; Santa Cruz, RN e Rio de Janeiro, RJ.

A participação dos idosos foi voluntária e foram respeitadas as regras da Comissão Nacional de Ética em Pesquisa do Ministério da Saúde (CONEPE-MS) para pesquisa com seres humanos. Eles foram recrutados em diversos locais - Programas de Saúde da Família, Unidades Básicas de Saúde, Centros de Convivência, amostras populacionais, Serviço Social do Comércio e uma operadora de saúde.

O Laboratório de Pesquisa em Envelhecimento Humano - GeronLab, da Universidade do Estado do Rio de Janeiro, liderou a investigação na cidade do Rio de Janeiro, constituindo o estudo Fragilidade em Idosos Brasileiros - seção Rio de Janeiro (FIBRA-RJ). De janeiro de 2009 a janeiro de 2010, portanto durante 13 meses, o FIBRA-RJ acompanhou os demais locais da rede FIBRA-BR, através de avaliações domiciliares de 847 indivíduos com 65 anos ou mais, residentes em bairros da zona norte do município do Rio de Janeiro, pertencentes ao cadastro de clientes de uma operadora de saúde. Porém, diferentemente das demais cidades da rede, esta avaliação constituiu a linha de base de uma coorte, em que foram conduzidas mais duas fases do estudo. A segunda teve início em julho de 2010, e foi concluída em junho de 2011; e a terceira, conduzida de agosto de 2012 a setembro de 2013. 
Este artigo tem como objetivo apresentar a metodologia de trabalho utilizada nas três fases do estudo de rede FIBRA-RJ.

\section{Métodos}

\section{Fase I}

\section{O protocolo de coleta de dados}

Nesta fase foi elaborado um questionário pela equipe de pesquisa envolvida no FIBRA-BR. A seleção amostral teve o principal objetivo de manter um painel suficiente a um acompanhamento de pelo menos quatro anos. Dentre as variáveis de interesse estavam questões que identificavam o estado mental, características sociodemográficas e saúde física autorrelatada, alterações no peso corporal, quedas, uso de medicamentos, uso de serviços de saúde, deficit de audição e de visão, hábitos de vida, aspectos funcionais da alimentação, capacidade funcional para atividades de vida diária, medidas de atividades físicas e antropométricas, avaliações da força muscular, da velocidade de marcha, autoeficácia de quedas, depressão, fadiga, e satisfação global com a vida. $\mathrm{O}$ protocolo de pesquisa pode ser obtido em www.geronlab.com.br

Os objetivos dessa fase foram analisar a prevalência de fragilidade pelos critérios propostos pelo Cardiovascular Health Study (CHS) ${ }^{2}$ e fatores associados, e também determinar o perfil de risco de fragilidade e fatores a ele relacionados.

\section{O plano amostral e estratégia de seleção}

A amostra, estratificada segundo o cruzamento entre as variáveis faixa etária e sexo, formou dez estratos naturais de amostragem com indivíduos dos sexos masculino e feminino, assim dispostos: 65 a 74 anos, 75 a 84 anos, 85 a 94 anos, 95 a 99 anos, 100 ou mais anos.

Os estratos finais foram definidos de acordo com o número de pessoas que compuseram os estratos naturais, e a seleção foi dada para os estratos até 94 anos de idade, sendo os demais estratos (95 a 99 anos de idade e 100 ou mais) dados como estratos certos, ou seja, todas as pessoas que estavam nestas duas faixas etárias foram contatadas para a realização da pesquisa. Dessa forma, decidiu-se agrupar estas duas classes em um único estrato para cada sexo.

A amostra de pessoas em cada estrato foi obtida por meio de amostragem aleatória inversa em cada um deles. Este tipo de amostragem, proposta por Haldane ${ }^{6}$, consiste em selecionar quantas unidades forem necessárias para que um número prefixado de entrevista seja obtido. Esse método é chamado de amostragem inversa porque, diferente do processo de amostragem simples, onde é definido o número de domicílios a serem visitados, na tentativa de se obter entrevistas, define-se o número de entrevistas que de fato serão realizadas. ${ }^{7}$ A maior vantagem é a de tornar desnecessário o aumento do tamanho amostral calculado para compensar a taxa de não resposta, resultando em menor custo da coleta de dados.

No estudo FIBRA-RJ, esta estratégia de amostragem passou a ser conduzida quando se identificou que seria necessária uma quantidade muito grande de elementos de reposição em cada estrato para se atingir o tamanho calculado de amostra em cada um deles. Sem perda de precisão e confiabilidade, foi possível conduzir os procedimentos de campo até que a amostra total fosse obtida.

\section{O cálculo do tamanho amostral}

O tamanho da amostra foi calculado de forma que o coeficiente de variação do estimador de diferentes prospectos para fragilidade, em cada estrato natural, fosse de $15 \%$ para estimativas de proporção em torno de 0,07 , com nível de confiança de $95 \%$. A amostra de indivíduos em cada estrato final foi obtida por meio de amostragem aleatória inversa em cada um deles. $\mathrm{O}$ tamanho final da amostra foi obtido pela soma dos tamanhos da amostra, calculados de cada um dos estratos. Em cada estrato, o número de pessoas sorteadas ( $\mathrm{nE}$ ) foi calculado a partir da fórmula: onde:

$$
n_{E}=\frac{(1,96)^{2} \cdot N_{E}^{2} \cdot S_{E}^{2}}{\left(2 \cdot C V_{E}^{2}\right) \cdot Y_{E}^{2} \cdot N_{E}^{2} \cdot S_{E}^{2}}
$$

$\mathrm{n}_{\mathrm{E}}$ é o tamanho populacional no estrato E;

$\mathrm{S}_{\mathrm{E}}$ é o desvio populacional associado ao estimador no estrato E;

$\mathrm{Y}_{\mathrm{E}}$ é o total populacional associado ao estimador no estrato E;

$\mathrm{CV}_{\mathrm{E}}$ é o coeficiente de variação prefixado associado ao estimador no estrato E.

Os tamanhos de amostra em cada estrato, ou $n_{E^{\prime}}$ foram inicialmente calculados para garantir as características já citadas de precisão das estimativas. A tabela 1 apresenta os totais de indivíduos por estrato e o número inicialmente selecionado e efetivamente consultado para obter o total estipulado em cada grupo. O total de indivíduos listados em cada estrato foi o necessário para se atingir o tamanho inicial da amostra estabelecido 


\section{Artigo original}

em cada um deles.

A amostra total para este estudo totalizou 900 indivíduos e os dados da tabela 1 apresentam o número de indivíduos selecionados por estrato (sexo versus faixa etária). Informam também o número total de registros enviados (2.640) para o preenchimento dos 900 questionários. Foi necessário um volume maior de informações devido à taxa de mortalidade nos grupos e da alta taxa de recusa associada às idades menores. Também, devido a problemas associados ao cadastro, um tratamento específico foi necessário para compor os quantitativos nos estratos.

A seleção e treinamento de auxiliares de pesquisa

Foram selecionados alunos de cursos de graduação de universidades públicas e privadas, além de profissionais interessados em atuar na área de pesquisa. $\mathrm{O}$ treinamento consistiu na leitura de textos sobre envelhecimento de forma a colocar o auxiliar de pesquisa em contato com a população a ser estudada. Isto feito, foram introduzidos os conceitos de pesquisa e repassado todo o manual de coleta de dados com informações detalhadas sobre o protocolo. Quando os auxiliares estavam complemente informados sobre o contexto da pesquisa, passaram ao treinamento de aplicação de instrumentos de avaliação funcional e cognitiva, além dos instrumentos de aferição de medidas antropométricas. Uma vez calibrados nesta etapa, os auxiliares foram treinados para o recrutamento da amostra, atividade realizada por telefone. $\mathrm{E}$, finalmente, o treinamento em controle de qualidade, armazenamento de dados em um banco específico e organização de planilhas. Durante o treinamento os supervisores observaram e avaliaram o desempenho dos auxiliares de pesquisa em cada tarefa para ao final dividi-los nas tarefas conforme a demonstração de maior habilidade. O processo de treinamento e organização de equipe teve a duração de seis meses, perfazendo um total de 480 horas.

\section{A organização da equipe de pesquisa}

A equipe de pesquisa apresentou a seguinte configuração: a) supervisores de campo - responsáveis por acompanhar todo o processo de trabalho, identificando os problemas e sugerindo e adotando medidas de resolução; b) recrutadores - equipe encarregada de fazer o contato telefônico com a amostra selecionada para agendamento da entrevista no domicílio e organização de planilhas com todo o histórico do recrutamento; c) auxiliares de campo - grupo destinado a realizar as visitas domiciliares para a aplicação do protocolo de coleta de dados; d) equipe de controle de qualidade responsáveis por receber os questionários de campo e fazer toda a revisão junto ao entrevistador de forma a não deixar questões geradoras de dúvidas no protocolo, diminuindo a chance de dados faltantes; $d$ ) digitadores - responsáveis pelo armazenamento de dados.

\section{O recrutamento da amostra}

Antes do início do recrutamento foram enviadas cartas para todos aqueles que foram sorteados para

Tabela 1. Totais populacionais por faixa etária e sexo, bem como tamanho das amostras em cada estrato $\left(n_{\mathrm{E}}\right)$.

\begin{tabular}{|c|c|c|c|c|}
\hline & Total populacional (NE) & $\begin{array}{l}\text { Tamanho inicial da } \\
\text { amostra (nE) }\end{array}$ & $\begin{array}{l}\text { Número de pessoas lista- } \\
\text { das em cada estrato }\left({ }^{*}\right)\end{array}$ & $\begin{array}{l}\text { Percentual de cada es- } \\
\text { trato do total de listados } \\
\text { para a amostra (\%) }\end{array}$ \\
\hline Homens (anos) & \multicolumn{4}{|c|}{ Frequência (n) } \\
\hline $65-74$ & 1381 & 108 & 361 & 13,7 \\
\hline $75-84$ & 1364 & 106 & 348 & 13,2 \\
\hline $85-94$ & 396 & 31 & 132 & 5,0 \\
\hline$\geq 95$ & 26 & 26 & 26 & 1,0 \\
\hline Mulheres (anos) & \multicolumn{4}{|c|}{ Frequência (n) } \\
\hline $65-74$ & 2688 & 210 & 649 & 24,6 \\
\hline $75-84$ & 2810 & 219 & 733 & 27,8 \\
\hline $85-94$ & 981 & 77 & 268 & 10,2 \\
\hline$\geq 95$ & 123 & 123 & 123 & 4,7 \\
\hline Total & 9769 & 900 & 2640 & 100,0 \\
\hline
\end{tabular}


participar da pesquisa informando sobre a parceria da universidade com a operadora de saúde a qual eram afiliados, bem como sobre os procedimentos de pesquisa. Três semanas após o envio das cartas, os recrutadores fizeram contato telefônico com o idoso objetivando o agendamento de entrevistas nos domicílios. Foram realizadas até oito tentativas telefônicas para encontrar o idoso, sujeito da pesquisa, ou o responsável pelo domicílio no caso de incapacidade do próprio idoso. Os contatos foram feitos em horários comerciais. Encontrando dificuldade neste processo, uma equipe de recrutadores realizou contatos telefônicos em horários noturnos e nos finais de semana até que fosse encontrada a pessoa. Nos casos onde os telefones estavam com defeito ou não se atendiam as ligações, um recrutador ficou responsável por ir diretamente ao domicílio do idoso para convidá-lo ou para se certificar que a pessoa não estava disponível.

Para analisar a prevalência de fragilidade, utilizaram-se os cinco itens da escala de fragilidade do CHS. (Tabela 2)

Para determinar o perfil de risco de fragilidade foi também utilizado o instrumento probabilidade de internação repetida (Pir) ${ }^{8}$ composto por oito itens incluídos no questionário: (a) autoavaliação da saúde com as seguintes opções de resposta: "muito boa, boa, regular, ruim ou muito ruim"; (b) internação no último ano; (c) número de consultas médicas no último ano; (d) presença de diabetes mellitus; (e) presença de doença coronariana; (f) sexo; (g) disponibilidade de um cuidador em caso de necessidade; (h) idade. A equação logística e os coeficientes de regressão correspondentes a cada um dos oito itens foram descritos por Pacala e colaboradores 9 . Os idosos foram estratificados como de alto risco quando o Pir era maior ou igual a 0,5 . O uso da fórmula encontra-se detalhado em www.geronlab.com.br

\section{Fase II}

A segunda fase do estudo teve início em julho de 2010, quando esta amostra foi novamente contatada com os objetivos de estimar a prevalência de demência em idosos da zona norte do Rio de Janeiro, validar o Informant Questionnaire on Cognitive Decline in the Elderly (IQCODE) $)^{10}$ e estudar algumas propriedades psicométricas do Questionário de Atividades Funcionais (QAF) de Pfeffer, 1982.11 Nesta etapa, 23 indivíduos foram excluídos por estarem institucionalizados, hospitalizados ou por mudança de endereço, 55 haviam falecido, 69 se recusaram a participar, 17 não foram localizados e 683 indivíduos foram analisados.

\section{O recrutamento da amostra}

Foram treinados cinco auxiliares de pesquisa para o recrutamento telefônico. As chamadas foram feitas inicialmente no horário das $8 \mathrm{~h}$ às $17 \mathrm{~h}$ de segunda a sexta-

\section{Tabela 2. Escala de fragilidade proposta pelo Cardiovascular Health Study e respectivos critérios diagnósticos.}

\begin{tabular}{|c|c|}
\hline Item & Medida \\
\hline Perda de peso & $\begin{array}{l}\text { Foram considerados como casos aqueles com autorrelato de perda não intencional de mais de } \\
4,5 \mathrm{~kg} \text { no ano anterior e/ou aqueles com } \mathrm{IMC}<18,5 \mathrm{~kg} / \mathrm{m}^{2} \text {. }\end{array}$ \\
\hline Força muscular & $\begin{array}{l}\text { Através do dinamômetro manual no membro superior dominante, solicitando ao participante que, } \\
\text { por três vezes, exercesse a maior força possível. Foram considerados casos os indivíduos no primei- } \\
\text { ro quintil após ajuste do resultado por sexo e IMC. }\end{array}$ \\
\hline Sensação de exaustão & $\begin{array}{l}\text { Foram utilizadas duas perguntas do Center for Epidemiological Studies Depression Scale (CES-D)*. } \\
\text { Item sete: "Eu me sinto cansado em tudo que faço" e item 20: "Não posso continuar desta forma". } \\
\text { Aqueles que responderam "sim" para qualquer das duas perguntas foram considerados casos. }\end{array}$ \\
\hline Lentificação da marcha & $\begin{array}{l}\text { Avaliado o tempo gasto para caminhar um percurso de } 4,6 \mathrm{~m} \text { em linha reta e, após ajuste para altu- } \\
\text { ra e sexo, foram considerados casos aqueles do primeiro quintil. }\end{array}$ \\
\hline $\begin{array}{l}\text { Diminuição da atividade } \\
\text { física }\end{array}$ & $\begin{array}{l}\text { Foi utilizado o questionário Minessota Leisure Time Activities (MLTA) e para cada atividade exerci- } \\
\text { da pelo idoso foi calculado o gasto calórico semanal. Foram considerados casos aqueles do primeiro } \\
\text { quintil. }\end{array}$ \\
\hline \multicolumn{2}{|c|}{$\begin{array}{l}\text { Fried LP, Tangen CM, Walston J, et al. Frailty in older adults: evidence for a phenotype. J Gerontol A Biol Sci Med Sci. } \\
\text { 2001;56(3):M146-56. }\end{array}$} \\
\hline \multicolumn{2}{|c|}{$\begin{array}{l}\text { *Batistoni SS, Neri AL, Cupertino AP. [Validity of the Center for Epidemiological Studies Depression Scale among Brazilian elder- } \\
\text { ly]. Rev Saude Publica. 2007;41(4):598-605. }\end{array}$} \\
\hline \multicolumn{2}{|c|}{$\begin{array}{l}\text { IlLustosa LP. Tradução e adaptação transcultural do Minnesota Leisure time physical activities questionnair em idosos. Geriatria } \\
\text { \& Gerontologia. } 2011 ; 5(2): 5 \text {. }\end{array}$} \\
\hline
\end{tabular}




\section{Artigo original}

feira. No caso de insucessos, novas tentativas de contato foram feitas nos fins de semana e à noite. Em uma breve ligação telefônica, convidava-se o idoso a participar, após explicar-se o objetivo da pesquisa e os procedimentos da coleta de dados. No caso de concordância por parte do participante, uma entrevista era agendada em uma unidade ambulatorial da universidade. Foram consideradas perdas aqueles indivíduos com os quais não se conseguiu contato após oito tentativas, no horário comercial, e mais quatro tentativas feitas à noite e/ou aos fins de semana.

Nesta etapa do trabalho foi solicitado ao idoso que indicasse um parente ou amigo que pudesse responder a algumas perguntas por telefone. Uma vez indicada a pessoa, o recrutador fazia contato por telefone e aplicava o QAF.11,12

\section{Seleção da amostra}

Nesta fase do estudo a amostra foi selecionada tomando por base o estado cognitivo e funcional dos indivíduos, buscando aqueles com suspeita de síndrome demencial. Para rastreio de declínio cognitivo foi utilizado o escore do Mini Exame do Estado Mental (MEEM) $)^{13,14}$ aplicado na fase 1 do estudo. Para rastreio de incapacidade funcional, após autorização do participante, o QAF11,12 foi aplicado a informantes próximos que conheciam o desempenho do idoso nas atividades de vida diária. Foram considerados elegíveis para avaliação clínica e neurocognitiva subsequentes os indivíduos cujo desempenho no MEEM e no QAF foi menor que 28 e maior ou igual a 5 , respectivamente.

Duzentos e cinquenta indivíduos foram considerados não portadores de disfunção cognitiva por terem desempenho no MEEM acima daquele ponto de corte. Outros 215 indivíduos também foram assim classificados, pois apesar de seus escores no MEEM terem sido menor que 28, tinham capacidade funcional preservada, isto é, QAF menor que 5. Duzentos e setenta e um indivíduos preencheram os critérios de suspeição de síndrome demencial, e foram convidados para a etapa posterior.

\section{Avaliação clínica e neurocognitiva da amostra}

Estado cognitivo global, capacidade funcional e humor

Os indivíduos tiveram o seu estado cognitivo global reavaliado pelo MEEM. ${ }^{14}$ Determinou-se a capacidade funcional com os seguintes instrumentos: a) atividades básicas da vida diária - $\mathrm{ABVD} ;{ }^{15}$ b) atividades instrumentais da vida diária - AIVD; ${ }^{16} \mathrm{c}$ ) inventário de atividades avançadas de vida diária - AAVD. ${ }^{17} \mathrm{~A}$ escala de depressão geriátrica $(E D G)^{18}$ e o Structured Clinical Interview for DSM-IV (SCID-DSM-IV) ${ }^{19}$ foram utilizados para avaliar alterações características de humor depressivo.

\section{Avaliação clínica}

Todos os indivíduos que aceitaram participar do estudo foram submetidos a uma avaliação clínica no serviço de Geriatria de uma unidade ambulatorial da Universidade do Estado do Rio de Janeiro. O foco desta avaliação foi a investigação da síndrome demencial. Para tal foi realizado exame físico com ênfase nos achados neurológicos, anamnese clínica com entrevista voltada para a avaliação do início e progressão das queixas de esquecimento, alterações de comportamento, sintomas depressivos, alterações da função renal e da função hepática, e solicitação de exames laboratoriais - hemograma completo, VDRL, HIV, Interleucina 6, vitamina B12, ácido fólico e hormônios tireoidianos.

Nesta etapa houve 55 recusas. Os idosos com diagnóstico de síndrome demencial foram encaminhados à ressonância magnética do crânio. Dos 105 encaminhados para este procedimento, 64 aceitaram e foram submetidos ao exame proposto pelo protocolo do estudo; 6 apresentaram resultado de ressonância magnética realizada no último ano; 10 recusaram-se e 25 apresentavam critérios que impossibilitavam a realização do mesmo, como prótese metálica e claustrofobia.

\section{Avaliação neurocognitiva}

Após a avaliação clínica, o idoso que não preencheu os critérios de exclusão para a realização dos testes - prejuízos graves de acuidade sensorial e demência em fase avançada - foi encaminhado para a avaliação neuropsicológica, que constou dos seguintes testes: a) teste de aprendizagem auditivo-verbal de Rey; ${ }^{20} \mathrm{~b}$ ) teste de fluência verbal FAS; ${ }^{21} \mathrm{c}$ ) subteste de habilidades visuoconstrutivas da bateria Mattis Dementia Rating Scale; ${ }^{22,23} \mathrm{~d}$ ) e os subtestes de raciocínio matricial, dígitos, códigos, compreensão e vocabulário da Wechsler Adult Intelligence Scale-III. ${ }^{24,25}$

\section{O diagnóstico da demência}

O diagnóstico sindrômico de demência foi estabelecido em consenso, por três médicos geriatras e um neuropsicólogo, de acordo com os critérios do Diagnostic and Statistical Manual of Mental Disorders - DSM-IV. ${ }^{19}$ Para o diagnóstico etiológico da síndrome demencial, além do DSM-IV, foram utilizados os consensos das seguintes instituições e grupos: National Institute of Neurological Communicative Disorder 
and Stroke - Alzheimer's Disease and Related Disorders Association - NINCDS - ADRDA; ${ }^{26}$ National Institute of Neurological Disorders and Stroke and the Association Internationale pour la Recherche et l'Ènseignement en Neurosciences - NINDS-AIREN. ${ }^{27}$

A trajetória dos indivíduos durante esta fase do estudo encontra-se detalhada na figura 1.

\section{Fase III}

A fase III foi realizada de agosto de 2012 a setembro de 2013, e teve os seguintes objetivos: estimar a prevalência e a incidência de sarcopenia em idosos residentes na zona norte da cidade do Rio de Janeiro; avaliar a validade preditiva do instrumento de rastreio de fragilidade "Probabilidade de internações repetidas".

\section{Recrutamento da amostra}

Assim como na fase II, auxiliares de pesquisa realizaram o recrutamento. Inicialmente, foi realizada uma abordagem por telefone para definir um contato pessoal para realização da entrevista e, em seguida, o idoso ou um informante adequado respondeu um questionário, cujo objetivo foi analisar o uso de serviços de saúde. Quando os indivíduos foram a óbito no período de seguimento entre as fases, perguntas específicas sobre esse desfecho foram incluídas na entrevista com o informante. O contato telefônico foi feito diariamente por bolsistas de iniciação científica, treinados para a execução da tarefa. Foram realizadas até oito tentativas até a localização do indivíduo. Nos casos de dificuldade de contato telefônico foram adotadas as seguintes es-

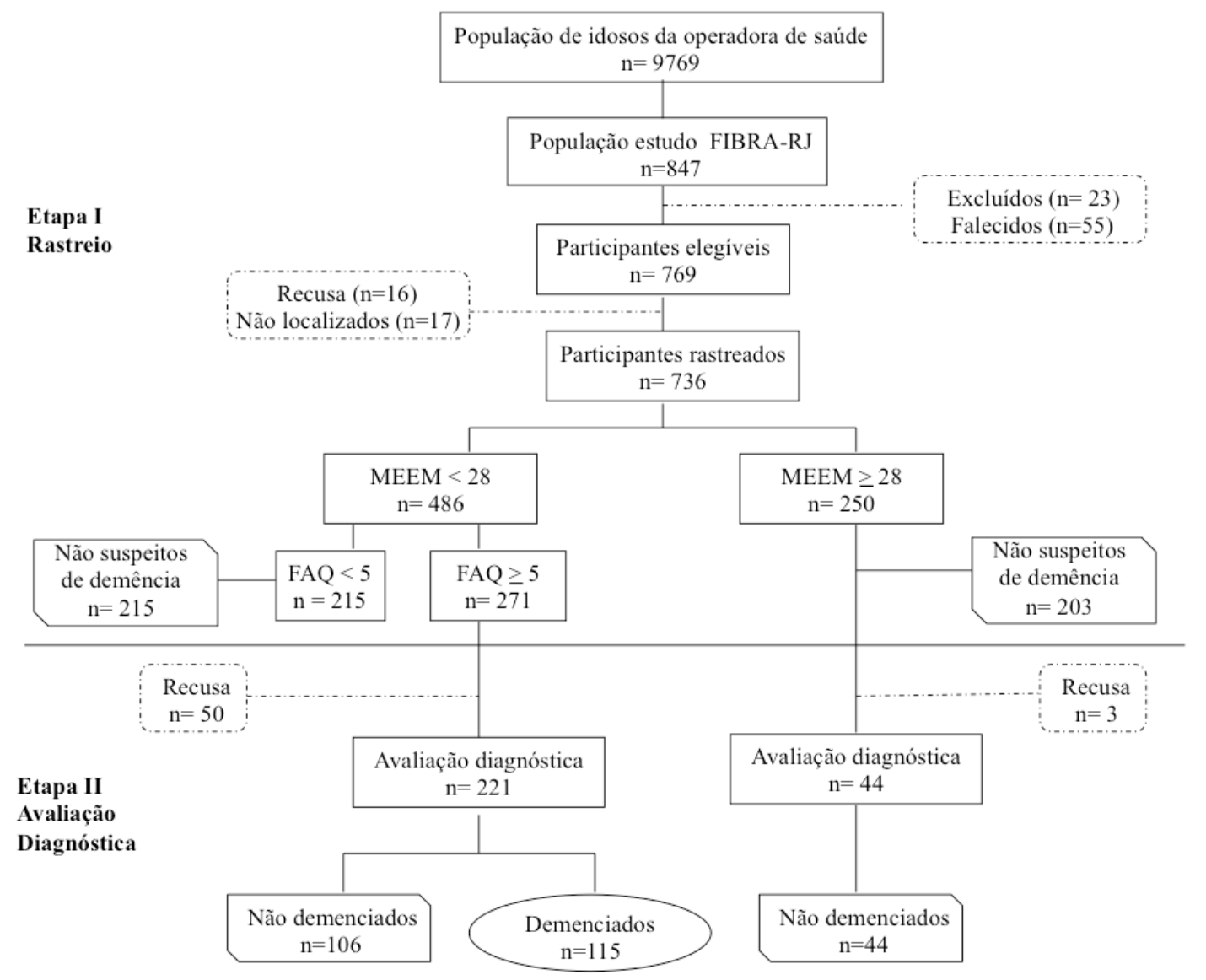

$\bigcirc$ Demenciados $\square$ Não demenciados $\quad$ Não avaliados

MEEM: Mini-Exame do Estado Mental; FAQ: Functional Activities Questionnaire

Figura 1. Diagrama de seleção da amostra FIBRA-RJ, Fase II. 


\section{Artigo original}

tratégias: a) contato telefônico com pessoas próximas para localizar os idosos; b) visitas no domicílio; c) visitas em domicílios de vizinhos de idosos, para tentar localizá-los, quando os mesmos não foram encontrados no endereço de origem. Foi considerado como perda o insucesso em localizar o idoso ou um informante substituto após todas as tentativas.

Nesta fase, tentou-se contatar os 847 indivíduos da linha de base. Dentre esses, 136 foram a óbito, 64 não foram localizados, 142 se recusaram e 103 foram excluídos por algum dos seguintes motivos: MEEM menor ou igual a 13; institucionalização; dificuldade de deambular; ou familiares informaram alteração cognitiva grave. A amostra final foi composta por 402 indivíduos, que foram entrevistados em seus domicílios, sendo reaplicado o questionário da primeira fase do estudo. Todos foram convidados a participar das etapas subsequentes, quando foram realizados os exames de sangue e a aferição da composição corporal. Para aqueles com dificuldade de deslocamento do seu domicílio até o local dos exames, foi oferecido transporte. Trezentos individuos aceitaram prosseguir com o estudo; 297 realizaram o exame de sangue; 284 rea- lizaram bioimpedância elétrica (BIA) e absorciometria de feixe duplo (DEXA) para avaliação da composição corporal (Figura 2). Aqueles que não realizaram os referidos exames foram excluídos por alguma condição que impediu o procedimento.

\section{Coleta de dados}

Após o agendamento da entrevista no domicílio o idoso recebeu a visita de um aluno de iniciação científica que, imediatamente após a leitura do termo de consentimento livre e esclarecido, aplicou o questionário, aferiu as medidas antropométricas e realizou a avaliação da marcha. Ao término da entrevista foi entregue um prospecto educativo com informações sobre sarcopenia, e foi informado ao idoso que em um momento futuro ele seria convidado para a realização de outros exames que ajudariam no diagnóstico do problema investigado. Esta etapa foi denominada primeiro deslocamento e deu sequência a mais três deslocamentos até o final da fase III do estudo FIBRA-RJ.

No segundo deslocamento os idosos foram convidados, por telefone, para comparecer no serviço de geriatria da Policlínica Piquet Carneiro, Universidade

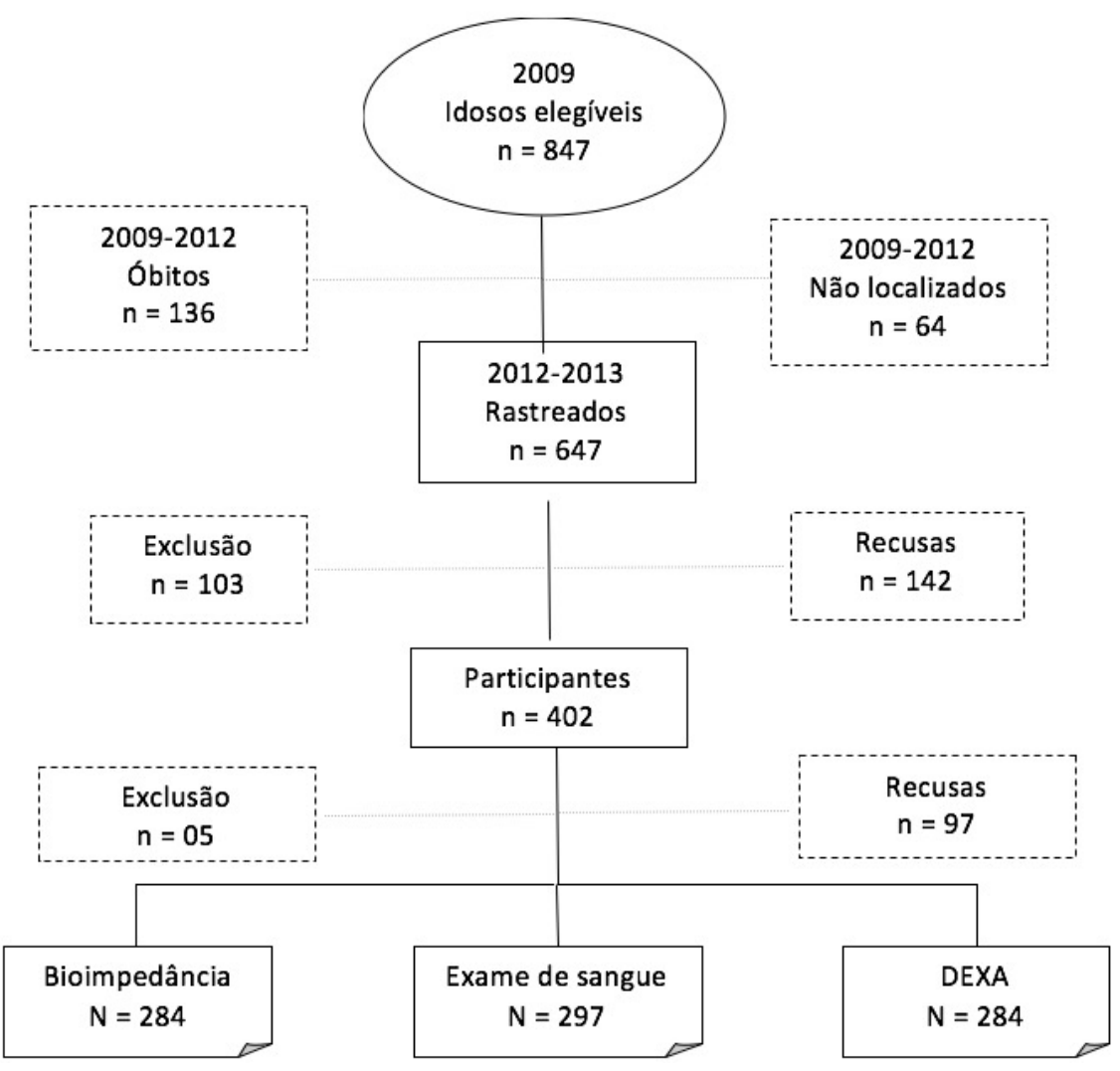

Figura 2. Diagrama de seleção da amostra FIBRA-RJ Fase III. 
do Estado do Rio de Janeiro, para a realização de exame de sangue, após jejum de 12 horas, e para a avaliação da massa muscular por bioimpedância elétrica. Antes de cada atividade os participantes liam e assinavam o Termo de Consentimento Livre e Esclarecido. Após esta etapa, foram convidados a participar da avaliação da composição corporal por DEXA.

Avaliação da composição corporal - BIA

A massa muscular foi estimada por BIA, utilizando um aparelho eletrônico, portátil, de impedância bioelétrica, visor de cristal líquido, corrente de baixa intensidade de $800 \mu \mathrm{A}$ (microampères), à frequência de $50 \mathrm{kHz}$, modelo QUANTUM II, marca RJL Systems, Inc. (USA).

Para realizar o exame, todos os idosos receberam orientações de consumir dois litros de líquidos no dia anterior ao teste; fazer jejum de, no mínimo, quatro horas; não praticar atividade física 24 horas antes do exame; evitar o uso de álcool 48 horas antes; evitar o uso de medicamentos diuréticos no dia anterior; urinar pelo menos 30 minutos antes da medida; e evitar o consumo de cafeína 24 horas antes do teste.

Avaliação da composição corporal - DEXA

Para a avaliação de composição corporal foi também utilizado o aparelho DEXA, modelo GE Lunar IDXA, de marca General Eletric. Este tipo de avaliação consiste na emissão de raios-X em duas frequências de intensidades diferentes, que ao passarem através do corpo do participante, separam-se por diferentes capacidades de atenuação dos raios-X nos tecidos de gordura e ósseo.

Para a realização do exame os participantes foram posicionados em decúbito dorsal sobre o equipamento, de forma a permanecerem totalmente centralizados em relação às laterais da mesa.

\section{Resultados}

O estudo FIBRA-RJ, deu origem a uma série de artigos, dissertações de mestrado e teses de doutorado.,12,28-36

\section{Discussão}

O estudo FIBRA-BR, por seu delineamento multicêntrico, provavelmente representou um dos esforços mais abrangentes de pesquisadores brasileiros, para a compreensão da dimensão da fragilidade e seus fatores associados, em território nacional.

O segmento Rio de Janeiro desse estudo, o FIBRA-RJ, representou uma tentativa de ir além dos objetivos inicialmente traçados, e dar continuidade ao acompanhamento da amostra examinada, investigando outros aspectos de acometimento de saúde, bastante comuns entre a população idosa, e ainda carentes de estudos populacionais com um número substancial de indivíduos.

Nas três fases que, até o momento, constituíram o FIBRA-RJ predominou o foco em estudos transversais descritivos da prevalência e fatores associados à fragilidade $^{3}$, síndrome demencial ${ }^{33}$ e sarcopenia. Além disso, alguns instrumentos de avaliação geriátrica foram abordados do ponto de vista da sua adaptação transcultural para o Brasil. 12,35,36

Se tais estudos têm conhecida limitação, associada à causalidade reversa, que impede conclusões seguras acerca da ordem cronológica dos fenômenos, no entanto, eram necessários, uma vez que, até então, poucos trabalhos descreviam tais condições na população brasileira.

Ainda, há que se ressaltar que os dados acumulados nos têm permitido refletir acerca de questões de extrema importância, tanto para a assistência ao idoso, quanto para a investigação da área de envelhecimento humano: mobilidade, massa muscular e força muscular.

Uma parte substancial das aferições utilizadas em Geriatria foram desenvolvidas por pesquisadores de países afluentes, validando-as nas suas populações. A velocidade de marcha usual e a força de preensão manual podem ser enquadradas entre estas medidas. São tão importantes na avaliação de idosos, que a força de preensão manual já é referida na literatura científica como o $6^{0}$ sinal vital. ${ }^{37}$ No entanto, em nosso meio, ainda não gozam desta popularidade e, tanto investigadores quanto clínicos, quando as usam, se vêm obrigados a definir a normalidade do fenômeno, baseados em pontos de corte desenvolvidos em outras realidades, na suposição não testada de uma universalidade improvável. Nossas conclusões, ainda que preliminares, vão em sentido oposto a tal universalidade, sugerindo que, para a população brasileira - e, provavelmente, para a latino-americana - outros valores de referência precisam ser definidos. ${ }^{38} \mathrm{~A}$ fase III nos permitirá estudar estas questões, estabelecendo a acurácia destas e outras aferições disponíveis no banco de dados, vis-à-vis mortalidade, desfechos adversos e uso de serviços de saúde.

A coorte FIBRA-RJ é oriunda da base de dados de uma operadora privada de serviços de saúde. Esta população de estudo, quando comparada com o conjunto de idosos brasileiros, tem uma maior renda, mais alta escolaridade e maior percentual de indivíduos nas faixas etárias mais velhas. Assim, os achados apresentados podem não ser comparáveis aos de estudos brasileiros 


\section{Artigo original}

de base populacional. Por outro lado, este perfil pode representar adequadamente uma parcela substancial da população idosa brasileira, isto é, aquela que é cliente dos serviços de saúde privados conveniados de operadoras de saúde, principalmente, no contexto dos grandes centros urbanos.

Além disto, o estudo de idosos com suporte do setor de saúde suplementar pode auxiliar na compreensão do impacto do acesso aos serviços de saúde na população mais velha. Este fato é relevante, pois as investigações epidemiológicas no Brasil conduzidas com as bases de registros de saúde, em geral, priorizam o setor público, negligenciando informações relevantes advindas de fontes como as operadoras privadas de saúde. A despeito destas limitações, as especificidades da amostra FIBRA-RJ podem representar grandes avanços no conhecimento sobre os agravos de saúde que acometem a população em idade avançada, agregando conhecimento sobre esta população, em geral, ainda pouco estudada no contexto brasileiro.

\section{Referências}

1. Campbell AJ, Buchner DM. Unstable disability and the fluctuations of frailty. Age Ageing. 1997;26(4):315-8.

2. Fried LP, Tangen CM, Walston J, et al. Frailty in older adults: evidence for a phenotype. J Gerontol A Biol Sci Med Sci. 2001;56(3):M146-56.

3. Moreira VG, Lourenco RA. Prevalence and factors associated with frailty in an older population from the city of Rio de Janeiro, Brazil: the FIBRA-RJ Study. Clinics (Sao Paulo). 2013;68(7):979-85.

4. Walston JD, Bandeen-Roche K. Frailty: a tale of two concepts. BMC Med. 2015;13:185.

5. Sundermann SH, Dademasch A, Seifert B, et al. Frailty is a predictor of short- and mid-term mortality after elective cardiac surgery independently of age. Interact Cardiovasc Thorac Surg. 2014 May;18(5):580-5. doi: 10.1093/icvts/ivu006. Epub 2014 Feb 3.

6. Haldane JB. On a method of estimating frequencies. Biometrika. 1945;33:222-5.

7. Vasconcellos MT, Silva PL, Szwarcwald CL. Sampling design for the World Health Survey in Brazil. Cad Saude Publica. 2005;21 Suppl:89-99.

8. Boult C, Dowd B, McCaffrey D, Boult L, et al. Screening elders for risk of hospital admission. J Am Geriatr Soc. 1993;41(8):811-7.

9. Pacala JT, Boult C, Boult L. Predictive validity of a questionnaire that identifies older persons at risk for hospital admission. J Am Geriatr Soc. 1995;43(4):374-7.

10. Jorm AF, Korten AE. Assessment of cognitive decline in the elderly by informant interview. Br J Psychiatry. 1988;152:209-13.

11. Pfeffer RI, Kurosaki TT, Harrah $\mathrm{CH}$ Jr, et al. Measurement of functional activities in older adults in the community. J Gerontol. 1982;37(3):323-9.

12. Sanchez MAS, Correa PCR, Lourenço RA. Cross-cultural Adaptation of the "Functional Activities Questionnaire - FAQ" for use in Brazil. Dementia \& Neuropsychologia. 2011;5(4):322-7.
13. Folstein MF, Folstein SE, McHugh PR. "Mini-mental state". A practical method for grading the cognitive state of patients for the clinician. J Psychiatr Res. 1975;12(3):189-98.

14. Lourenco RA, Veras RP. Mini-Mental State Examination: psychometric characteristics in elderly outpatients. Rev Saude Publica. 2006;40(4):712-9.

15. Lino VT, Pereira SR, Camacho LA, et al. Cross-cultural adaptation of the Independence in Activities of Daily Living Index (Katz Index). Cad Saude Publica. 2008;24(1):103-12.

16. Lawton MP, Brody EM. Assessment of older people: self-maintaining and instrumental activities of daily living. Gerontologist. 1969;9(3):179-86.

17. Reuben DB, Laliberte L, Hiris J, et al. A hierarchical exercise scale to measure function at the Advanced Activities of Daily Living (AADL) level. J Am Geriatr Soc. 1990;38(8):855-61.

18. Paradela EM, Lourenco RA, Veras RP. Validation of geriatric depression scale in a general outpatient clinic. Rev Saude Publica. 2005;39(6):918-23.

19. Association AP. Diagnostic and statistical manual of mental disorders: DSM-IV-TR. Washington DC: American Psychiatric Pub Inc; 2000.

20. Rey A. L'Examen Clinique en Psychologie: Presse Universitaires de France; 1958.

21. Benton A. Multilingual Aphasia Examination. lowa City: AJA Associates, Inc; 1989.

22. Mattis S. Dementia rating scale (DRS). Odessa, FL: Psychological Assessment Reources; 1988.

23. Porto CS, Fichman HC, Caramelli $P$, et al. Brazilian version of the Mattis dementia rating scale: diagnosis of mild dementia in Alzheimer's disease. Arq Neuropsiquiatr. 2003;61(2B):339-45.

24. Wechsler D. WAIS-III administration and scoring manual. San Antonio, TX: The Psychological Corporation; 1997.

25. Nascimento E. Adaptação, validação e normatização do WAISIII para uma amostra Brasileira. In: Wechsler D, editor. WAIS-III: Manual para administração e avaliação. São Paulo: Casa do Psicólogo; 2004.

26. McKhann G, Drachman D, Folstein M, et al. Clinical diagnosis of Alzheimer's disease: report of the NINCDS-ADRDA Work Group under the auspices of Department of Health and Human Services Task Force on Alzheimer's Disease. Neurology. 1984;34(7):939-44.

27. Roman GC, Tatemichi TK, Erkinjuntti T, et al. Vascular dementia: diagnostic criteria for research studies. Report of the NINDS-AIREN International Workshop. Neurology. 1993;43(2):250-60.

28. Faria CA, Lourenco RA, Ribeiro PC, et al. Cognitive performance and frailty in older adults clients of a private health care plan. Rev Saude Publica. 2013;47(5):923-30.

29. Perez M, Lourenco RA. FIBRA-RJ Network: frailty and risk of hospitalization in the elderly in Rio de Janeiro, Brazil. Cad Saude Publica. 2013;29(7):1381-91.

30. Malini FM, Lourenco RA, Lopes CS. Prevalence of fear of falling in older adults, and its associations with clinical, functional and psychosocial factors: The Frailty in Brazilian Older People-Rio de Janeiro Study. Geriatr Gerontol Int. 2016 Mar;16(3):336-44. doi: 10.1111/ggi.12477. Epub 2015 Apr 14.

31. Faria CA, Lourenco RA, Ribeiro PC, et al. Cognitive performance and frailty in older adults clients of a private health care plan. Rev Saude Publica. 2013;47(5):923-30.

32. Correa Ribeiro PC, Lopes CS, Lourenco RA. Complexity of lifetime occupation and cognitive performance in old age. Occup Med (Lond). 2013;63(8):556-62. doi: 10.1093/occmed/kqt115. 
Roberto A. Lourenço e cols. • Fragilidade em Idosos Brasileiros - FIBRA-RJ: metodologia de pesquisa dos estudos de fragilidade, distúrbios cognitivos e sarcopenia

Epub 2013 Nov 9.

33. Correa Ribeiro PC, Souza Lopes C, Lourenco RA. Prevalence of dementia in elderly clients of a private health care plan: a study of the FIBRA-RJ, Brazil. Dement Geriatr Cogn Disord. 2013;35(1-2):77-86.

34. Sanchez MA, Lourenco RA. Screening for dementia: Brazilian version of the Informant Questionnaire on Cognitive Decline on the Elderly and its psychometric properties. Geriatr Gerontol Int. 2013;13(3):687-93.

35. Lourenco RA, Sanchez MA. Accuracy of the Brazilian version of the informant questionnaire on cognitive decline in the elderly at screening for dementia in community-dwelling elderly participants: findings from FIBRA-RJ study. J Geriatr Psychiatry Neurol. 2014;27(3):212-9.

36. Reichenheim M, Sanchez MA, Lourenco RA. Re-assessing the dimensional structure of the Informant Questionnaire on Cognitive Decline in the Elderly (IQCODE): empirical evidence for a shortened Brazilian version. BMC Geriatr. 2015;15:93.

37. Syddall H, Cooper C, Martin F, et al. Is grip strength a useful single marker of frailty? Age Ageing. 2003;32(6):650-6.

38. Lourenço RA, Pérez-Zepeda M, Gutiérrez-Robledo L, et al. Performance of the European Working Group on Sarcopenia in Older People algorithm in screening older adults for muscle mass assessment. Age Ageing. 2015;44(2):334-8. 